\title{
Self-organizing Behavior of Y-junctions of Graphene Nanoribbons
}

\author{
Dávid Fülep*, Ibolya Zsoldos* ${ }^{*}$ István László*** \\ * Faculty of Technology Sciences, Széchenyi István University, Hungary \\ ** Department of Mathematics and Computational Sciences, Széchenyi István University, Hungary \\ *** Department of Theoretical Physics, Budapest University of Technology and Economics, Hungary
}

\begin{abstract}
With the help of our molecular dynamics simulation we want to motivate emerging and development of technological methods for building of carbon nanostructure networks. We shall study self-organizing behaviors of graphene nanoribbons in Y-junctions. We determine the conditions for perfect formation of nanotube $\mathrm{Y}$ junctions from parallel nanoribbons. The role of graphene nanolithography in nanoribbon network and nanotube network production is studied. Our simulations show the possibility of nanotube network realization as well.
\end{abstract}

Keywords: molecular dynamics, graphene, nanoribbon, nanotube, network, simulation.

\section{INTRODUCTION}

The graphene as one graphite sheet is a zero-band gap semiconductor, which hinders its application as a semiconductor devise. The band structure turns to be more favorable if we cut out graphene ribbons from the sheet [1-3]. Depending on the orientation of the ribbon on the sheet, the band gap will be different of zero. The width of the band gap of armchair nanoribbons depends on the number of the carbon atoms modulo 3 in the cross direction. If the remainder is 0 or 1 the ribbon is semiconductor and 2 belongs to the zero-band gap in tight-binding calculation [4]. According to the theoretical calculations the zigzag ribbons are semiconductors due to the small band gap. According to the experiences the graphene ribbons are semiconductors [5]. After clarifying the electronic behaviors, it was proved that the graphene based structures can be building boxes of the nano size electronic devises in the future. The appropriate technological conditions are however missing. The electronic properties of the nanoribbons are very interesting but the experimental realizations are still not without problem. The present methods are based mostly on chemical and physical procedures. With the help of chemical methods Yang et al. constructed graphene nanoribbons of length $12 \mathrm{~nm}$ from 1,4diiodo-2,3,5,6-tetraphenylbenzene and 4-bromophenylboronic acid molecules [6]. They were using various catalysts. Cai et al. connected to chains the precursor molecules with the help of active surfaces and after cyclic dehydrogenization they obtained nanoribbons of various widths [7]. According to the experiences these methods can not control the length, the width and the edges of the ribbons.

The physical methods are more promising concerning the accuracy [8]. Kosynkin et al. produced $100 \%$ perfect nanoribbons by cutting nanotubes with oxidative methods [9]. Jiao et al. were using plasma for cutting up multi wall carbon nanotubes [10]. The tailoring methods of the graphene sheets are named lithographic methods. The simplest lithographic method applies an STM needle to move or brake the graphene sheet [11]. In the other methods a voltage is applied between the AFM needle and the graphene sheet. By changing the voltage and by moving the needle the graphene can be oxidized and various patterns can be cut out from the graphene [12-14].

In other lithographic methods etching is used with nanoparticles in diffusion processes in gases. The present most precise lithographic method is the carbothermic etching (CTE) [18], where in the first step artificial defects are produced on a graphene/ $\mathrm{SiO}_{2}$ support system. In this method not only ribbons but predefined patterns, as Y-junction ribbons can be produced. With this method even a ribbon of $2.5 \mathrm{~nm}$ can be produced. In our days this is the most precise lithographic method for producing nanoribbons.

As there is missing only an order of magnitude for obtaining atomic precise nanopatterns, theoretical calculations were motivated to simulate the formation of 3D nanostructures from graphene patterns. It was proved in molecular dynamics calculations that C60 and C70 fullerene molecules can be formed from one graphene pattern in molecular dynamics simulations [19-21]. It was proved also that zigzag and armchair nanotubes can be obtained from graphene patterns in molecular dynamics simulations. As the driving force was generated with 
the help of a half fullerene the final tube was closed with a half fullerene at one end [21].

The production of nanotubes of two nanoribbons is important because in this way open ended nanotubes can be produced [21]. The production of nanotubes from two parallel nanoribbons is not a trivial task. First the possibility was published [22-23], and after that the topological and energetic conditions for obtaining perfect tubes were published [24]. It is well known that the nanotubes have very interesting electronic and mechanical properties. They are more stable than the ribbons and they are also suitable for interesting electronic nanodevices. As the nanotubes do not have such kind of dangling bonds as the ribbons, they do not have the instability at edges. From nanotubes more stable structures can be built as from the ribbons. The production of nanotubes with given properties is not yet solved. As the graphene tailoring with atomic precision will be possible, the graphene lithography of nanotube production from parallel graphene ribbons can make possible at the same time the production of networks for nanotubes and nanoribbons as well. In nanotube networks the Y-junctions play the principal role beside the straight tubes [25]. Thus, the study of Y-junctions has basic important beside that of the straight tubes. In this paper we study the formation of nanotube $\mathrm{Y}$ junctions from graphene ribbons. We examine whether the topological and energetic conditions obtained for the nanotube formation are sufficient or not in the case of more complicated structures, like $\mathrm{Y}$-junctions.

\section{THE METHOD}

We have studied the formation of $\mathrm{Y}$ junctions in molecular dynamics (MD) [26-27] calculations where the initial structure was made of two Y-shaped graphene patterns cut out from the graphene sheet. The initial distance between the two patterns was $0.35 \mathrm{~nm}$. The carbon-carbon interaction was calculated with the help of Density Functional Theory adjusted Tight Binding method (DFT-TB) [28]. The time step was $\Delta \mathrm{t}=0.7$ fs and Verlet algorithm [29] was used in the calculation of the velocities of the carbon atoms. The environmental temperature was controlled with the help of NoséHoover thermostat [27-28, 30-31]. The application of the Nosé-Hoover thermostat made it possible that we could start our simulation with randomly determined initial velocities of the carbon atoms. The corresponding $\mathrm{T}_{\text {init }}$ atomic temperature was different of the environmental temperature of the simulation. This initial temperature was taken from the range [1000,1100] Kelvin. According to our experiences the final structure was not depending strongly by increasing or decreasing the temperatures with scaling. In contrast, it was found that the final results were influenced much more strongly when randomly changing the initial velocities.

In our previous publication [24, 32] we have studied the self-organized formation of nanotubes from graphene ribbons in molecular dynamics simulation. The distance between the two initial ribbons was $0.35 \mathrm{~nm}$. There we defined the $E_{c}=E_{\text {tube }}-E_{\text {graphene }}$ curvature energy of nanotubes, where $E_{\text {tube }}$ and $E_{\text {graphene }}$ are the formation energies of nanotube and graphene. We have found further a linear function between reciprocal square of the tube radius $R$ and the curvature energy: $E_{c}=a / R^{2}+b$. Here $E_{c}$ is in $e V$, the radius $R$ is in $\AA$ and $\mathrm{a}=2.4005 \mathrm{eV}^{2}$ and $\mathrm{b}=-0.0227 \mathrm{eV}$. Using this function for the curvature energy and applying several test calculations we defined critical ribbon widths under which there is a very small probability of nanotube formation from ribbons. These critical ribbon widths are in order $9.23 \AA$ and $15.99 \AA$ for the straight armchair and zigzag nanotubes.

We have found further that the application of support increases significantly the efficiency of nanotube formation. Beside this advantage the support has an another one as well. Namely it makes possible precise positioning of the patterns one over the other in a practical realization. Between the ribbon and the support, we supposed van der Waals interaction [32]. In a recent publication nanoribbon rings role up in a self-organizing way on metal wires [33]. Similar rolling up process was studied on nanotubes due to van der Waals interactions [34]. Self-assembly of fullerenes and graphene flakes were studied in molecular dynamics calculations [35]. Artificial defects on graphene ribbons [36] or tailoring of graphene flakes [37] also induced selfscrolling by the van der Waals interaction. Important role was played by van der Waals force in interaction of nano spheres [38] as well.

\section{BUILDING UP OF THE Y-JUNCTION IN A SELF-ORGANIZING MANNER FROM ONLY ONE GRAPHENE FORMATION}

In case of carbon nanotube $\mathrm{Y}$-junctions, in accordance with the topological conditions, the possibility of designing from a single graphene formation also emerges. As to, a junction consisting of closed-end tubes, can be designed topologically from a single formation [21]. The graphene formation that can be seen in Fig. 1.c, designed for a closed-end $\mathrm{Y}$-junction, is shown in Fig. 1.a. 


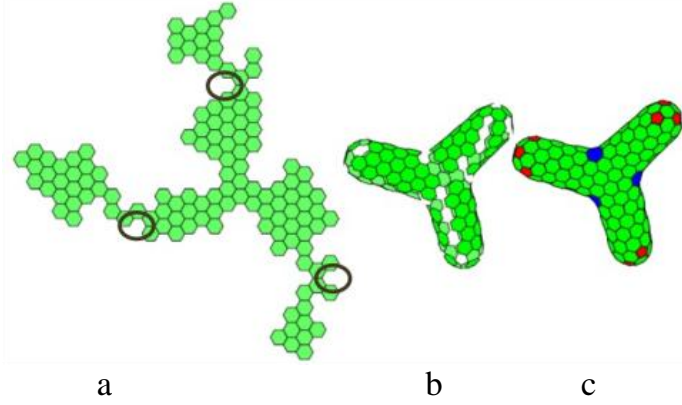

Fig. 1: Constructability of a closed end carbon nanotube $\mathrm{Y}$-junction from a sole graphene formation [21]. a: The model of a graphene formation for the construction of a carbon nanotube $\mathrm{Y}$-junction. b: A nanotube $\mathrm{Y}$-junction model made by the up-folding of the formation shown in Fig. a. c: The entire model of the derived nanotube Y-branch (pentagons and heptagons highlighted by different colors). The ellipses indicate the location of the formation of some pentagons, see below.

The formation is made up of hexagons clearly, and the number of vertices (carbon atoms) on both models are identical. If we wind up the graphene formation seen in Fig. 1.a, then according to Fig. 1.b we get the designed structure.

It is therefore legitimate to wonder whether the carbon nanotube Y-junctions can build up in a selforganizing way, from a single graphene formation? We expect that by the abandonment of the graphene formation, that can be seen in Fig. 1.a, bonds get established in the framed areas (the figure was not encumbered with the indication of all the occurrences), with the new bonds pentagons get into the structure, due to the pentagons the structure winds up and the proposed Y-junction could coalesce. For the examination of the question, on the graphene formation that can be seen in Fig. 1.a, we performed a molecular dynamics simulation. We have met several problems, due to which we could not build the planned $\mathrm{Y}$-junction from only one graphene formation.

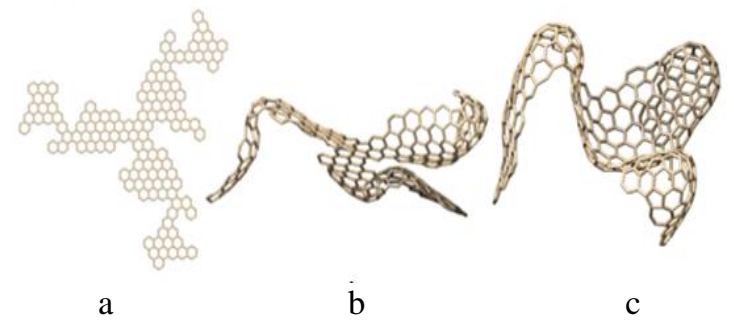

Fig. 2: Input model and molecural dynamics simulation results for the build-up of a carbon nanotube Y-juction from a single graphene formation. a: Another input model constructed similarly to Figure 1.a. b: The result of the MD simulation for the model of Fig. 1.a. c: The result of MD simulation for the model of Fig. 2.a.
The first problem is that the pentagons, created because the new chemical bonds, wind up the structure into different, unexpected directions, therefore the build-up of the Y-junction is impossible, as shown in Fig 2.b. Similarly to the graphene formation shown in Fig. 1.a, several other formations can be designed for the build-up of the same carbon nanotube Y-junction, we can see another example in Fig. 2.a. The other problem can be seen on the MD simulation run result of this new formation (Fig. 2. c): though, the formation started to wind up into the planned directions, in this case, near the centre, the structure went wrong due to unwanted coalescences. We have found that by varying the formations, after further experiments ending with similar results, for the flawless adhesion, taking place in a self-organizing manner, the building process must be carried out from an over-complicated graphene formation, there are too many possibilities for bending into different directions and for the coalescences in unexpected places.

\section{THE SELF-ORGANIZING BUILD-UP OF A Y-JUNCTION FROM PARALLEL GRAPHENE RIBBONS}

Because of the complexity of the structure of the only graphene formation, moreover, it is also justified to evolve open-end tubes, that we build the carbon nanotube Y-junctions by coalescence of parallel graphene ribbons. We studied the following topological cases:

- the models of the Y-junctions consisting of armchair nanotubes,

- the models of the Y-junctions consisting of zigzag nanotubes,

- heterogeneous Y-junction models, where, in the structure, there are armchair and zigzag nanotube branches as well.

In every case, we started from a model consisting of two parallel graphene ribbons [24]. The distance of the ribbons was equal to the equilibrium distance between the layers of the graphite crystals: $3.35 \AA$ (in case of the zigzag models, containing the substrates as well, we deviated from this, this will be indicated in the right place). We examined by molecular dynamics simulation that after the abandonment of the structure whether there are any new bonds forming between the ribbon edges, whether these two parallel graphene ribbons coalesce into a nanotube $\mathrm{Y}$-junction.

The dimensions have been chosen in order to conform to the earlier research results obtained for the straight carbon nanotubes [24]: Critical ribbon width, derived from curvature energy, distinguishes cases of low and high probability of perfect 
coalescence. This critical width is $9.23 \AA$ for armchair and $15.47 \AA$ for zigzag straight nanoribbons, as it was mentioned in the Introduction.

In case of the models of the Y-junctions we examined the critical ribbon width range. For the molecular dynamics simulation experiments we used models of three different widths from the range around critical width.

The topological cases of Y-junctions are examined in detail in the following chapters.

\subsection{Armchair-type Y-junctions, case without substrates}

In Fig. 3. input models can be seen that were prepared for the molecular dynamics simulation for the construction of $\mathrm{Y}$-junctions composed of armchair type nanotubes.

With molecular dynamics simulations, starting from ribbons that are narrower than the critical ribbon width, we did not get an immaculate structure. We have experienced that even if certain parts begin to build up faultlessly, the smoothing force form a graphene sheet spoils the structure in the other places.
In Fig. 4. the results of two independent runs are presented. The structure goes wrong in Fig. 4.a at two branches, and in Fig. 4.b at one branch so that the coalescence starts on one side only, after that the branch osculates into a graphene sheet instead of winding up and forming a tube. We performed both experiments on the model presented in Fig. 4.a.

Starting from ribbons of critical ribbon width or from ribbons the width of which is wider that the critical one, from the parallelly superposed graphene ribbons a perfect carbon nanotube $\mathrm{Y}$-junction was built. We present the results of the molecular dynamics simulation belonging to this in Figs. 5.a and 5.b.

Regarding the case of the armchair-type Y-junctions, based on the above experiments, it can be said that the tendency for straight carbon nanotubes prevails, that is to say, that after the abandonment in an equilibrium distance, the graphene ribbons of critical and of wider than critical width can coalesce into a flawless carbon nanotube $\mathrm{Y}$-junction in a selforganizing manner.

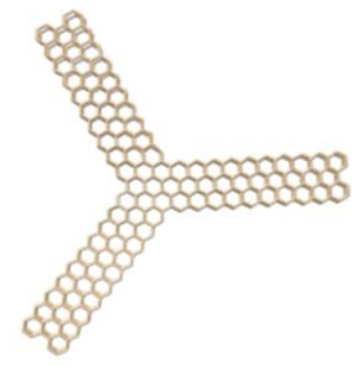

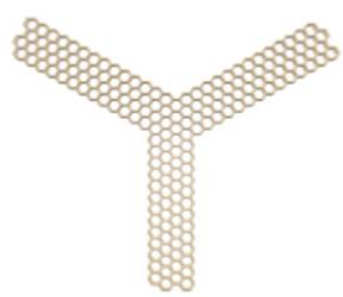

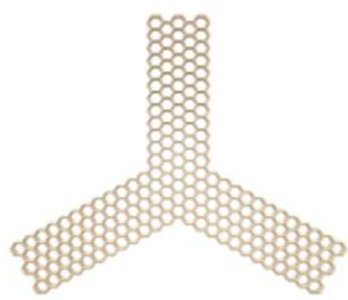

Fig. 3: Graphene nanoribbon models planned for the construction of armchair carbon nanotube Y-junctions. a: the width of the ribbons is less than the critical ribbon-width: $7.1 \AA$, b: the width of the ribbons is equal to the critical ribbon width: $9.23 \AA$, c: the width of the ribbons is more than the critical ribbon-width $11.36 \AA$.
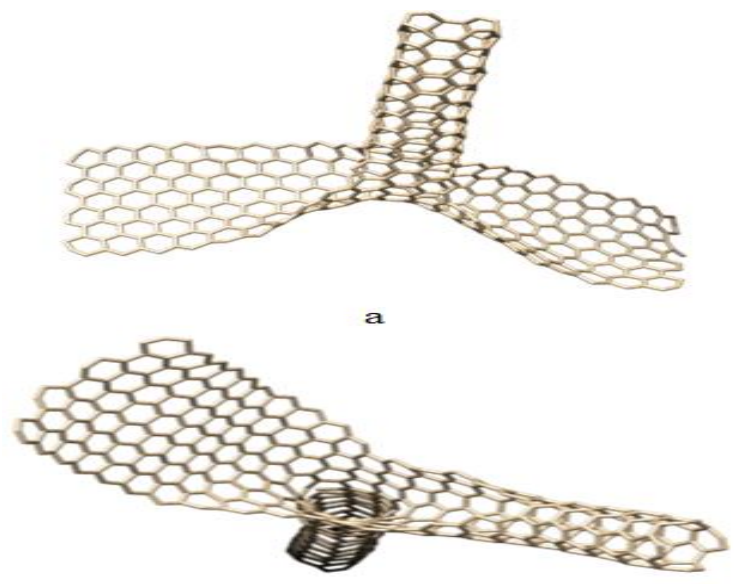

b

Fig. 4: The simulation results run on the model of Fig. 3.a for the construction of carbon nanotube Y-junctions. 

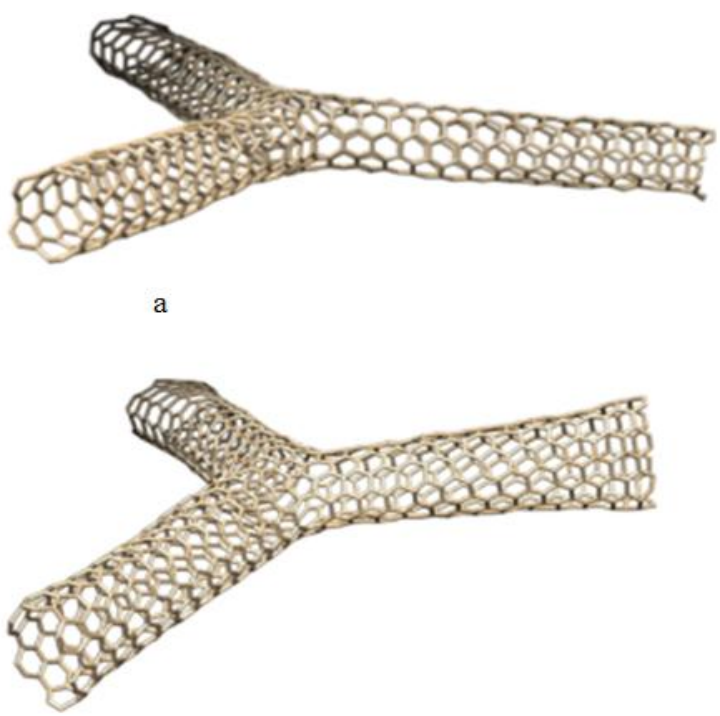

$\mathrm{b}$

Fig 5. a: Simulation result ran on the model of Figure 4.b for the construction of a carbon nanotube Y-junction. b: Simulation result ran on the model of Figure 4.c for the construction of a carbon nanotube Y-junction.

\subsection{Heterogeneous and zigzag type $\mathrm{Y}$-junctions, the case without substrates}

As heterogeneous carbon nanotube $\mathrm{Y}$ junctions, those structures are defined, between the branches of which both armchair-type and zigzagtype straight tubes are located. When we design an electronic network from $\mathrm{Y}$-junctions, in all likelihood, the heterogeneous junctions will be the most interesting ones, because, due to the different electrical behavior dependent on the crystalorientation, this is where there is a big chance for the construction of various electronic devices.

If we insert a zigzag branch into the structure, instead of one of the branches of the armchair graphene Y-junction presented in Fig. 6., we get a heterogeneous junction. On the example in Fig. 6. we present how the zigzag branch can be constructed between the armchair branches.

In Fig. 6. it can be seen that the atoms located at the edges of the zigzag branch are in a less favorable position in terms of adhesion than the atoms of the armchair branches:

- The atoms of the armchair ribbons are situated one above the other in an exact cover along the ribbon edges as well.

- This favorable position at the edges of the zigzag branch is topologically not allowed. One of the ribbons should be slightly wider so that at the coalescence the expected hexagon pattern can develop.

In Fig. 6., in the enlarged details of the edges, we labeled the distances between the atoms located on the edges with $d_{1}$ and $d_{2}$. We expected that there would be a bond established between the labeledpositioned atoms that are $d_{1}$, or $d_{2}$ distance away from each other and thereby the two parallel ribbons coalesce. In case of an equilibrium ribbon distance $(3.35 \AA) d_{1}=3.63 \AA, d_{2}=3.35 \AA$. Since $d_{1}>d_{2}$, as a consequence, the zigzag branch has less chance to coalesce than the armchair branches.

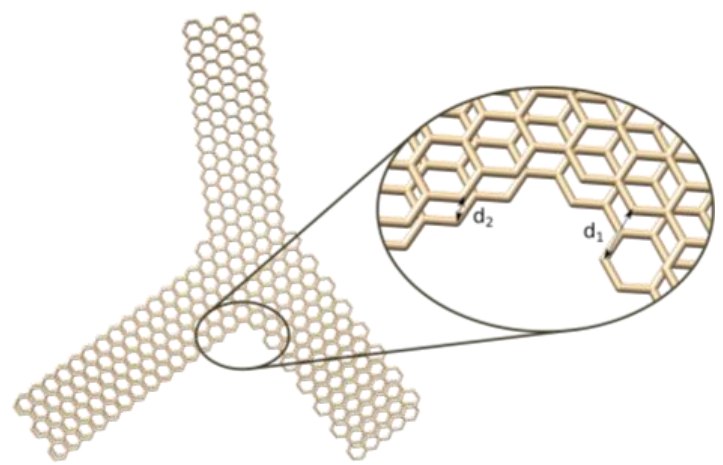

Fig. 6: Heterogeneous Y-junctions of parallel graphene ribbons in top view (the ribbons cover each other), as well as the enlarged detail of the meeting of two different branches of crystal-orientation. In the zigzag branch the upper ribbon along the circumference is smaller with one atom row than the lower ribbon, while the ribbons below each other at the armchair branches are of the same width.

From the molecular dynamics simulations, it has been found that the $\mathrm{Y}$-junctions of graphene ribbons, containing zigzag branches, abandoned in an equilibrium distance did not coalesce into a perfect carbon nanotube Y-junction in any of the tested ribbon- width cases. This result has also come out regarding the junctions comprising of purely zigzag junctions.

In Figs. 7. and 8. we show the examined graphene models: the heterogeneous junction models can be 
seen in Fig. 7. the models containing zigzag branches exclusively in Fig. 8. The ribbon widths, in case of all models, were chosen in a range close to the critical ribbon width.

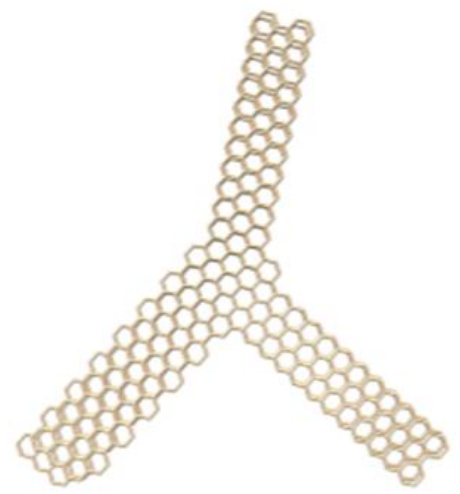

a

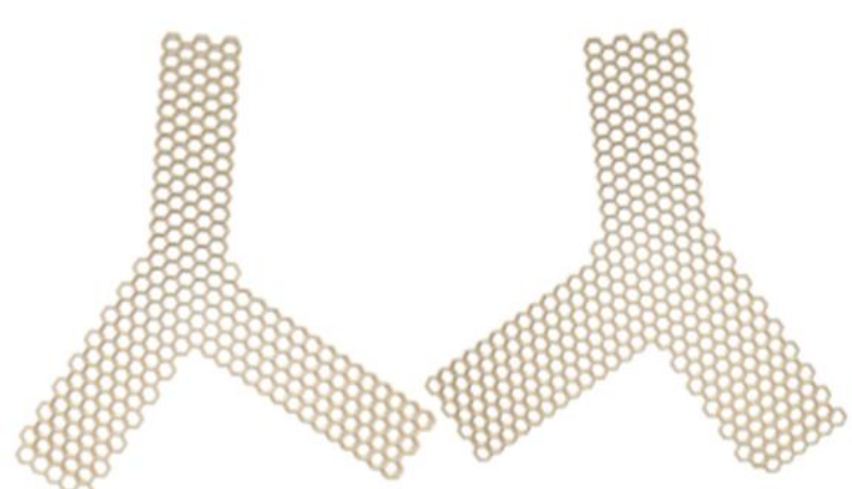

$\mathrm{b}$ c

Fig. 7: Heterogeneous Y-junction models. a: small-width (7.1 $\mathrm{A}, 7.1 \AA$, $8.61 \AA$ ), b: medium-width $(11.36 \AA$, $11.36 \AA$ and $11.07 \AA$ ), c: large-width $(13.0 \AA, 13.0 \AA$ and $15.99 \AA)$ models. At the armchair branches the ribbons that are located below each other have the same width and they are in exact cover, at the zigzag branch the top ribbon along the circumference is smaller with one atomic row than the lower ribbon.

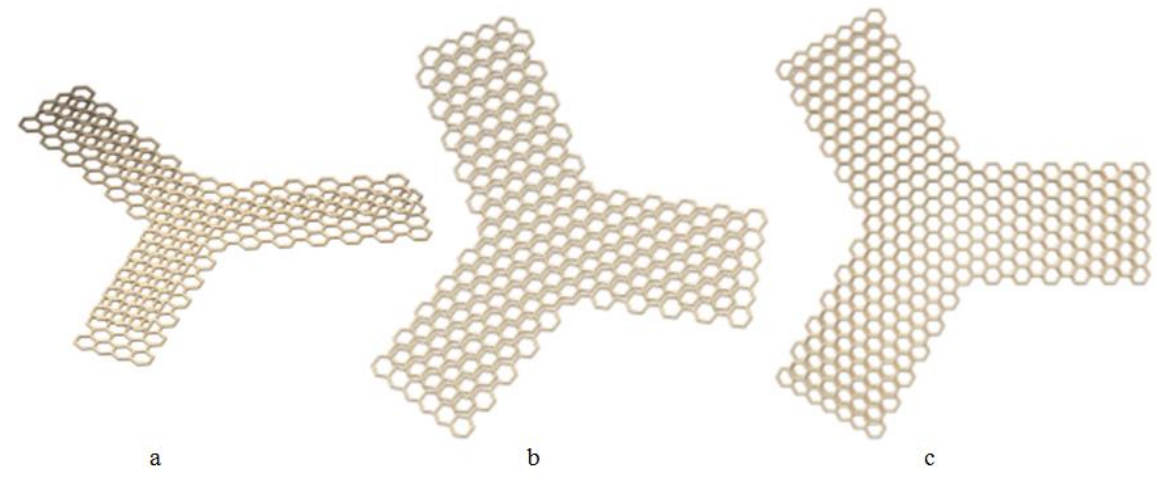

Fig. 8: Zigzag Y-junction models. The top ribbon along the circumference is one atomic row smaller than in case of the lower ribbon. a: small-width (7.38 $\AA$ and $9.84 \AA$ ), b: medium-width $(12.3 \AA$ and $14.76 \AA$ ), c: largewidth (14.76 ̊ and $17.22 \AA)$ models.

In Table 1 we summarized the MD simulation results of the graphene ribbon $\mathrm{Y}$ junctions abandoned at an equilibrium distance.

Overall it can be stated that for the armchair junctions, the tendency of flawless coalescence in the case of straight nanotubes has remained, however, as soon as only one zigzag branch gets into the structure it is unfavorable from the viewpoint of flawless coalescence: the possibility of a flawless self-organizing formation could not be justified with a successful run.

\begin{tabular}{|c|c|c|c|}
\hline $\begin{array}{l}\text { WITHOUT } \\
\text { SUBSTRATE }\end{array}$ & \multicolumn{3}{|c|}{$\begin{array}{c}\text { Self-organizing behavior of carbon nano-tube } \mathrm{Y} \text {-junctions with } \\
\text { models without substrates }\end{array}$} \\
\hline Size & Armchair & Heterogeneous & Zigzag \\
\hline small & (model of Fig. 3.a) & (model of Fig. 7.a) & (model of Fig. 8.a) \\
\hline medium & (model of Fig.3.b) & (model of Fig. 7.b) & (model of Fig. 8.b) \\
\hline large & (model of Fig.3.c) & (model of Fig. 7.c) & (model of Fig. 8.c) \\
\hline
\end{tabular}

Table 1: Running experiences of different sized and oriented carbon nanotube Y-junctions without substrate means: unsuccessful coalescence, $\quad$ means: perfect (flawless) coalescence. 
The unsuccessful coalescence of the heterogeneous Y-junctions could clearly be explained with the unfavorable position of the edges of the zigzag branches. In Fig. 9. some typical heterogeneous cases of the unsuccessful attempts are shown.

In Fig. 9.a the coalescence of one of the armchair branches has taken place properly, but only one side of the other armchair branch and zigzag branch has coalesced, the opposite side has remained open, and due to the opening up into a graphene sheet there was no chance to perform a perfect coalescence. In Fig. 7.b such runtime results are shown where the armchair branches have coalesced properly, but in the zigzag branch the coalescence has not even begun. Finally, the lesson to be learnt from the simulation results of Fig. 7.c is that the osculation of the zigzag branch into a graphene sheet did not allow the neighboring armchair branch to grow together either.

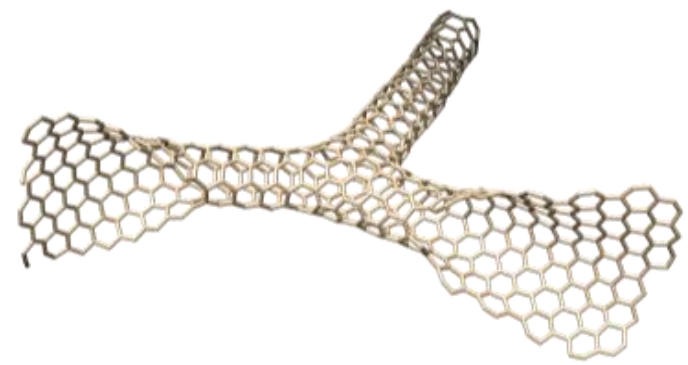

a

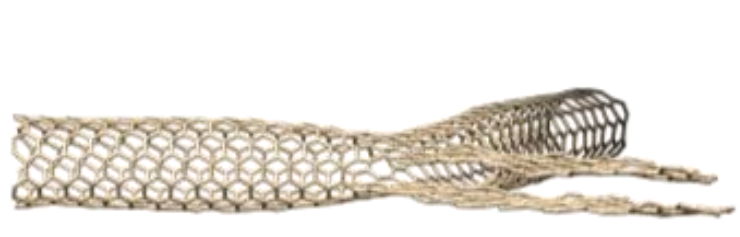

$\mathrm{b}$

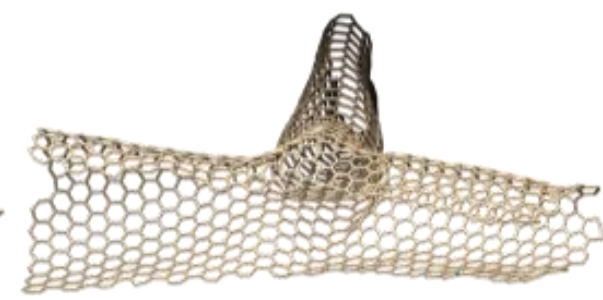

$\mathrm{C}$

Fig. 9: Typical cases of unsuccessful coalescence of (heterogeneous) graphene ribbon $Y$-junctions, containing zigzag branches. a: model consisting of $7.1 \AA$, 7.1 $\AA$ wide armchair and $8.61 \AA$ wide zigzag ribbons run on the model of Figure 7.a. b: model consisting of $11.36 \AA$, $11.36 \AA$ wide armchair and $11.07 \AA$ wide zigzag ribbons run on the model of Figure 7.b, c: zigzag model consisting of $13.0 \AA$, $13.0 \AA$ wide armchair and $15.99 \AA$ wide ribbons, run on the model of figure 7.c.

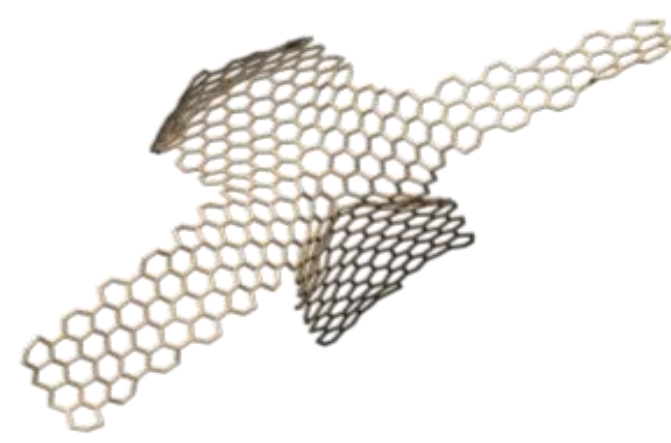

a

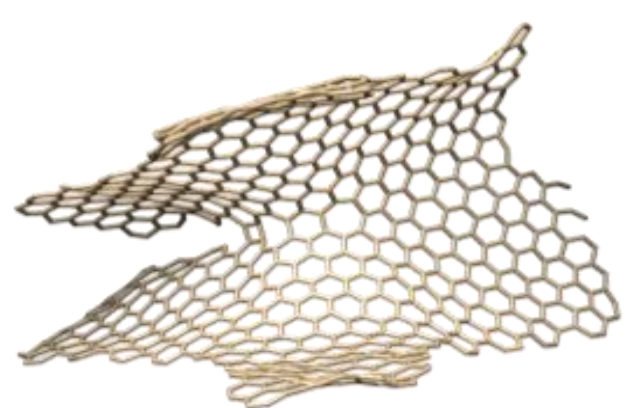

$\mathrm{b}$

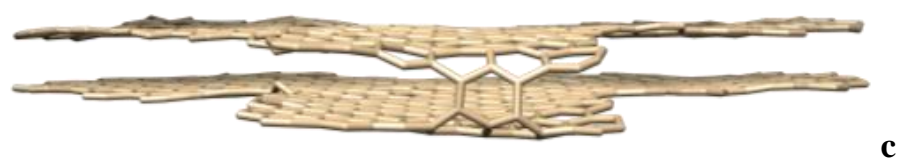

Fig 10:. Broken, zigzag Y-junctions without substrates. a: a model prepared based on Fig. 8.a, b: a model the size of which is identical to the size of Fig. 8.b, c: a model the size of which is identical to the size of Fig. 8.c.

In Fig. 10. the running results of the full zigzagbranch cases are presented. In case of the models of small and medium ribbon width, the coalescence has taken place on one or two sides, but the structure has opened up, see Fig. 10.a-b.
In case of the model of wider ribbons, after the slight displacement that took place due to the unfavorable speed directions, the structure has started to coalesce, however, instead of the sides, it happened at the ends of the ribbons, Fig. 10.c. 


\subsection{Coalescence of substrate-containing parallel graphene Y-junction models}

From the edifications of the unsuccessful runs illustrated in Fig. 9. and 10, we have found likely, that we can get significantly better results if the simulations are carried out with substrates. On models of straight carbon nanotubes, we have already succeeded in showing the beneficial effect of the substrates from the viewpoint of coalescence [32]. We thought that the substrate, as a physical constraint, will not allow, in case of the junctions either, the turning out of the half-coadunate sections abiding to the evolution of coercion into graphene sheet, moreover, it will not allow the graphene ribbons positioned one above the other to slip aside either.

In this case, as a substrate, similarly to the case of the straight ribbons [32], we have chosen a multi-layer graphene sheet, too. At the simulation input we placed the two parallel graphene ribbons between the substrates in accordance with the equilibrium distance characteristic to the crystal planes of the graphite crystal (see in Fig. 11), here we launched the runs also with the conditions of $\mathrm{d}_{\mathrm{A}}$ $=\mathrm{d}_{\mathrm{B}}=\mathrm{d}_{\mathrm{n}}=3.35 \AA$.

For the modeling of the van der Waals interaction between the substrates and the graphene nanoribbons, in the present case, we applied the Lennard-Jones potential as well, with the parameters used in case of straight tubes [32].

During the simulations, in case of models that also contain substrates, the structure was abandoned only after the bonds between the carbon atoms placed at the edges of the two graphene ribbons had already been established, that is, the structure was already coalesced.

In case of the models presented in Figs. 3., 7. and 8. we executed MD simulation runs. We have summarized the results obtained by resource to the substrate in Table 2. The content of Table 2 is worth to be compared with the data of Table 1 . We have presented, where and under what conditions the successful self-organizing build-up of 3D junctions seems possible. The problem regarding the zigzag branches, the role of the substrates and the necessity of compressing the substrates at the zigzag junctions can be seen clearly.

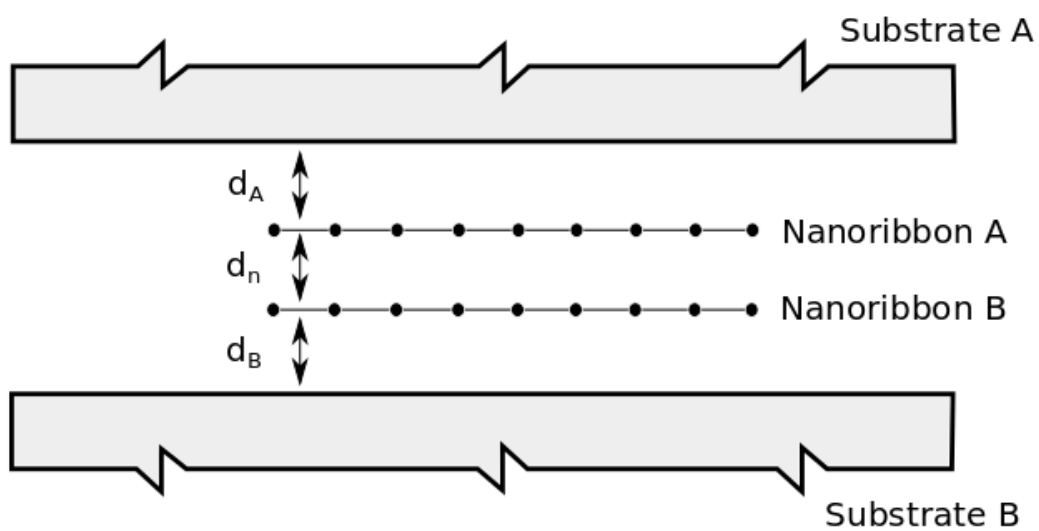

Fig. 11. Placement of nanoribbons between substrates (side view)

The results prove the important role of the substrates:

- In case of the armchair $Y$-junctions, even in the case of a ribbon width below the critical width, a perfect coalescence has been achieved.

- In case of the heterogeneous junctions, the simulation experiments also resulted in a perfect coalescence.

- As for the zigzag junctions, the first attempts have ended unsuccessfully in case of all sizes and we found defective coalescences even in the presence of substrates. When we started the simulation from the equilibrium distance between the parallel ribbons $\left(d_{n}=3.35 \AA\right)$ the structures have not coalesced perfectly due to the unfavorable positions of the edges of the zigzag branches, even in the presence of substrates. With additional runnings we have managed to show that if in case of the input model the equilibrium distance between the parallel ribbons is reduced to $d_{n}=3 \AA$ (namely the substrates, and thereby the ribbons of the input model are compressed) then, even in case of the zigzag junctions, a flawless coalescence is obtained during the MD simulations.

\begin{tabular}{|c|c|}
\hline $\begin{array}{c}\text { WITH } \\
\text { SUBSTRATE }\end{array}$ & $\begin{array}{c}\text { Self-organizing behavior of carbon nano-tube Y-junctions with } \\
\text { models containing substrates }\end{array}$ \\
\hline
\end{tabular}




\begin{tabular}{|c|c|c|c|}
\hline Size & TMArmchair & Heterogeneous & Zigzag \\
\hline small & $\begin{array}{c}\mathrm{d}_{\mathrm{n}}=3.35 \AA \\
\text { (model of Fig. 3.a) }\end{array}$ & $\begin{array}{c}\mathrm{d}_{\mathrm{n}}=3.35 \AA \\
\text { (model of Fig. 7.a) }\end{array}$ & $\begin{array}{c}\mathrm{d}_{\mathrm{n}}=3.35 \AA \\
\text { (model of Fig. 8.a) }\end{array}$ \\
\hline medium & $\begin{array}{c}\mathrm{d}_{\mathrm{n}}=3.35 \AA \\
\text { (model of Fig.3.b) }\end{array}$ & $\begin{array}{c}\mathrm{d}_{\mathrm{n}}=3.35 \AA \\
\text { (model of Fig. 7.b) }\end{array}$ & $\begin{array}{c}\mathrm{d}_{\mathrm{d}}=3.35 \AA \\
\text { (model of Fig. 8.b) }\end{array}$ \\
\hline large & $\begin{array}{c}\mathrm{d}_{\mathrm{n}}=3.35 \AA \\
\text { (model of Fig.3.c) }\end{array}$ & $\begin{array}{c}\mathrm{d}_{\mathrm{n}}=3.35 \AA \\
\text { (model of Fig. 7.c) }\end{array}$ & $\begin{array}{c}\mathrm{d}_{\mathrm{d}}=3.35 \AA \\
\text { (model of Fig. 8.c) }\end{array}$ \\
\hline
\end{tabular}

Table 2: Experiences about the running of Y-junctions of different sizes and orientation, containing substrates. means: unsuccessful coalescence, $\checkmark$ means: perfect coalescence.

In Fig. 12 the runtime results of the armchair, while in Fig. 13 the runtime results of the heterogeneous junctions are shown. In case of both orientation models, in case of all three dimensions, perfectly coalesced nanotube Y-junctions were obtained, therefore the self-organizing formation is possible.

In Fig. 14. we present the results of the MD simulations launched on the models of the ribbons containing only zigzag branches, with the help of substrates from an equilibrium $\left(\mathrm{d}_{\mathrm{n}}=3.35 \AA\right)$ distance. Beside the positive effect of the substrates, uncertainties still have remained in the system: the slight sliding of the ribbons, one above the other, have made the models predisposed to initiate the coalescence at the end of the ribbons instead of at the edges. Basically, this was the reason why all the models have gone wrong.

In Fig. 15. the results of the MD simulations launched on the models of only zigzag ribbons can be seen, with the help of substrates from a distance less $\left(d_{n}=3.00 \AA\right)$ than the equilibrium $\left(d_{n}\right.$ $=3.35 \AA$ ) distance. From the ribbons compressed between the substrates, in case of all three dimensions, flawlessly coalesced nanotube Yjunction was obtained.

\section{CONCLUSION}

According to our molecular dynamics simulations the topological and energetic conditions of successful nanotube formation from nanoribbons are not completely valid in Y-junction cases. At the equilibrium distance of $3.35 \AA$ they are valid only in the case of armchair cases. Only one zigzag nanotube in any branch of the junction can destroy the perfect coalescence of the Y patterns. With the help of the support the number of favorable junction formations increases significantly even in the case of zigzag tubes in any branch. We obtained perfect coalescence in the case of only zigzag nanotubes in each branch by decreasing the initial equilibrium distance between the patterns from $3.35 \AA$ to $3 \AA$. 


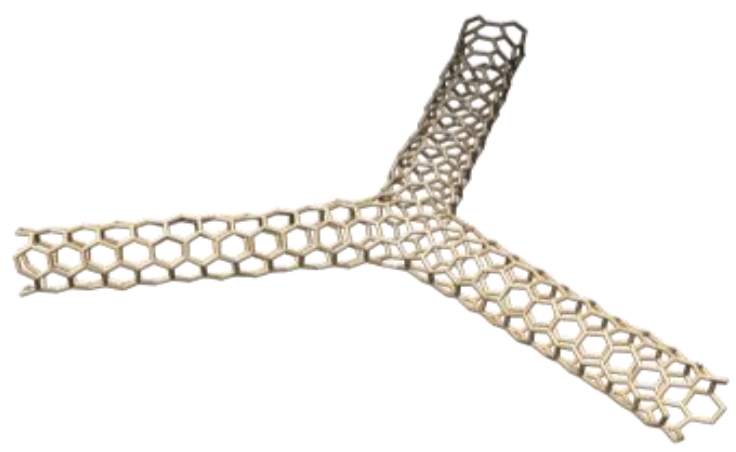

a

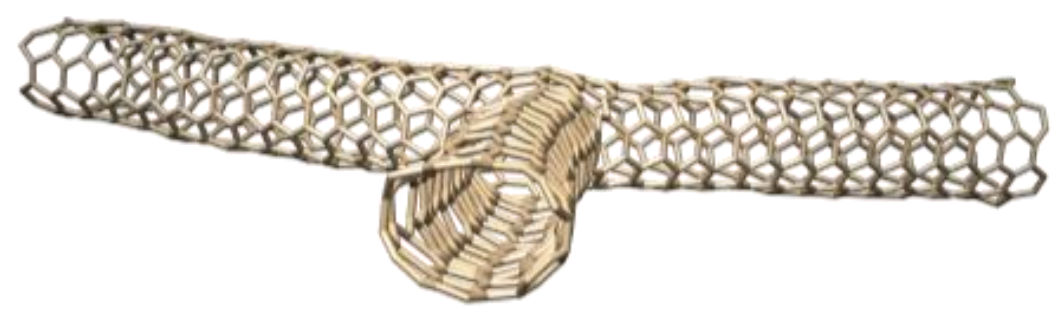

b

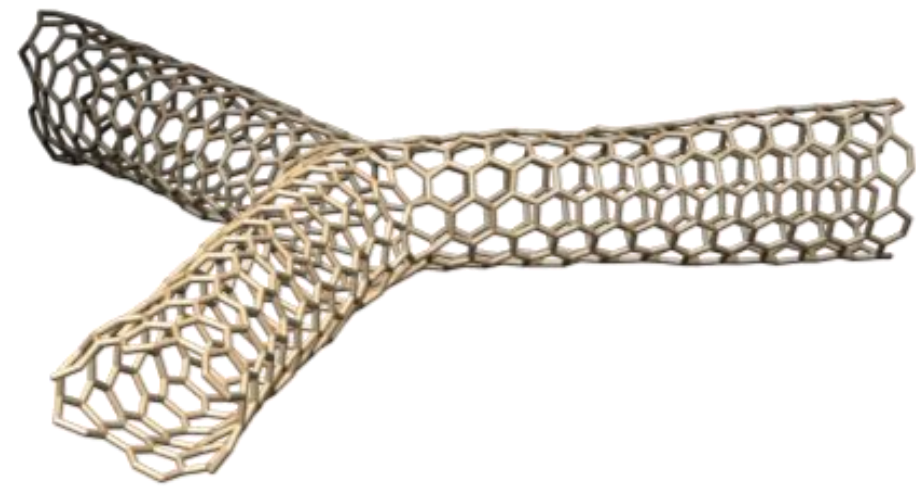

$\mathrm{c}$

Fig. 12: Models of successful armchair Y-junctions grown with substrates a: the result of the MD simulation run on the model of Fig. 3.a, b: the result of the MD simulation run on the model of Fig. 3.b, c: the result of the MD simulation run on the model of Fig. 3.c.

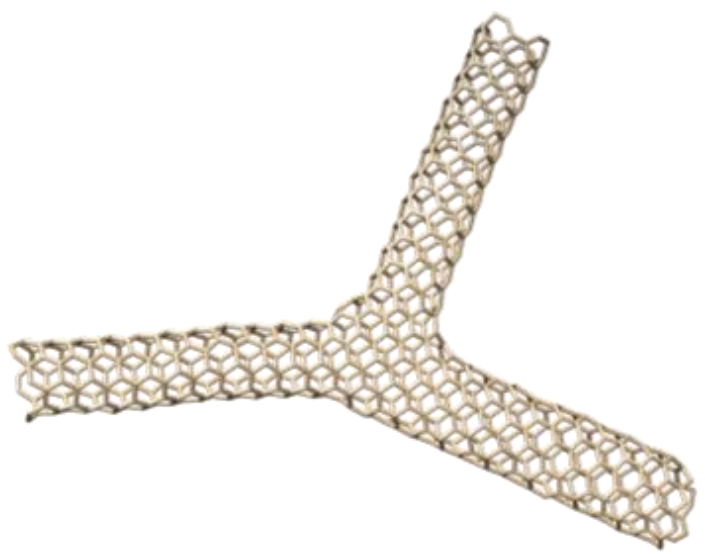

a 


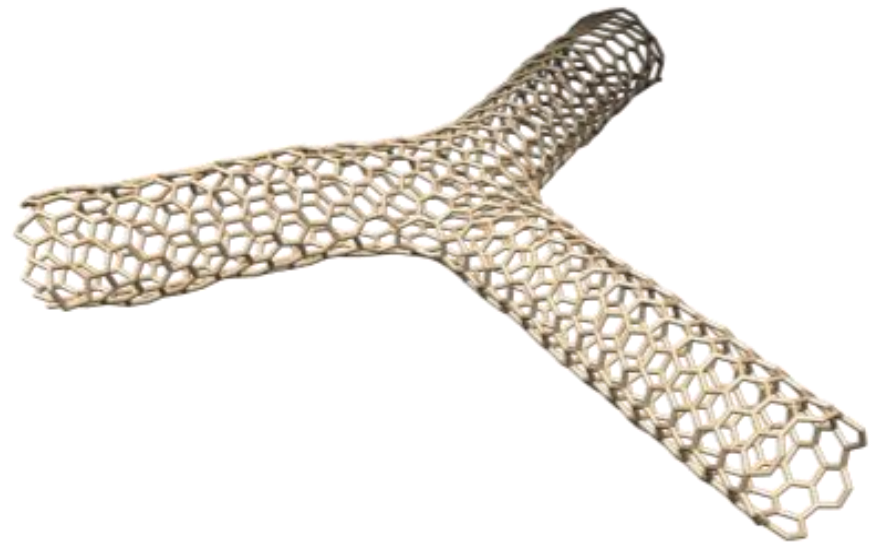

b

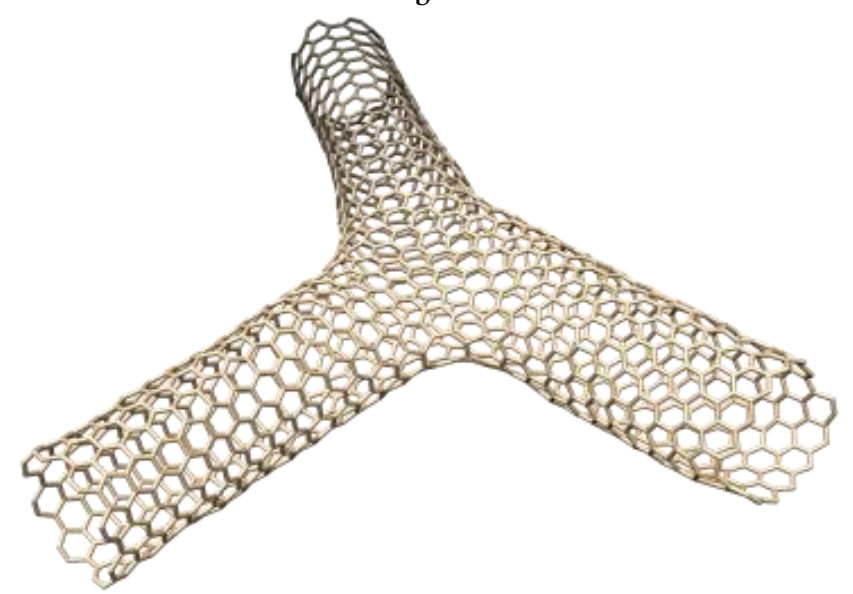

c

Fig. 13: Models of successful heterogeneous $Y$-junctions grown with substrates. a: the result of the MD simulation run on the model of Fig. 7.a, b: the result of the MD simulation run on the model of Fig. 7.b, c: the result of the MD simulation run on the model of Fig. 7.c

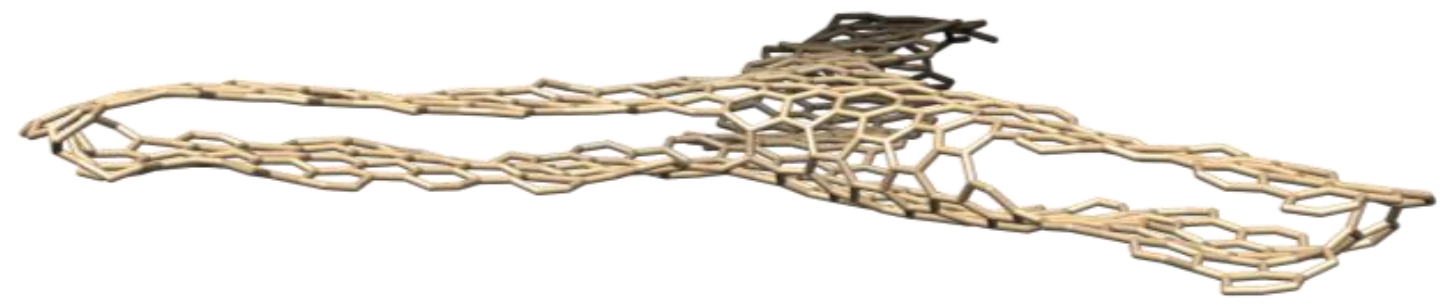

a

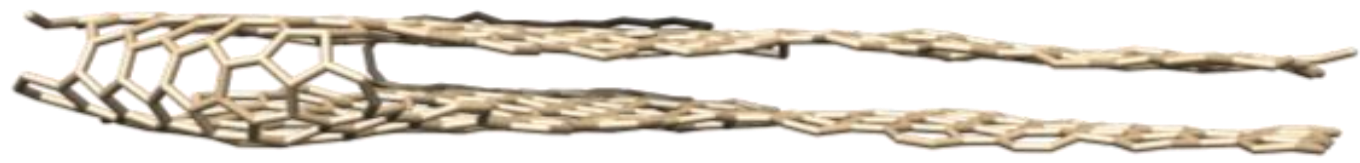

b

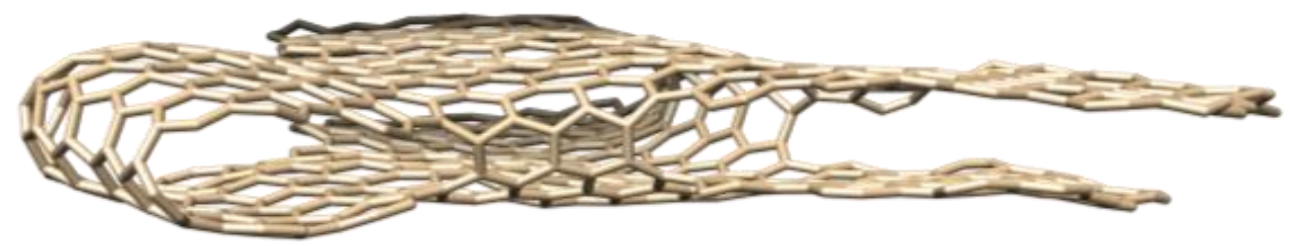

$\mathrm{c}$ 
Fig. 14: The models of unsuccessful zigzag Y-junctions with substrates, after the running of an MD simulation from an equilibrium $(\mathrm{dn}=3.35 \AA$ ) distance. a: the result of the MD simulation run on the model of Fig. 8.a, b: the result of the MD simulation run on the model of Fig. 8.b, c: the result of the MD simulation run on the model of Fig. 8.c.

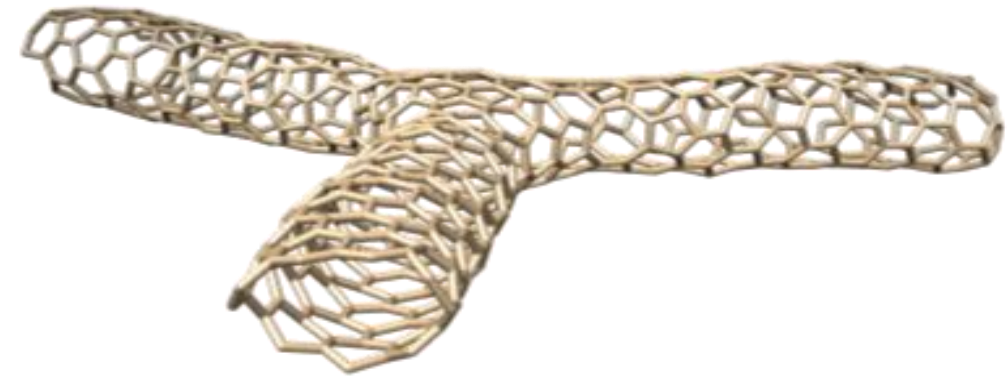

a

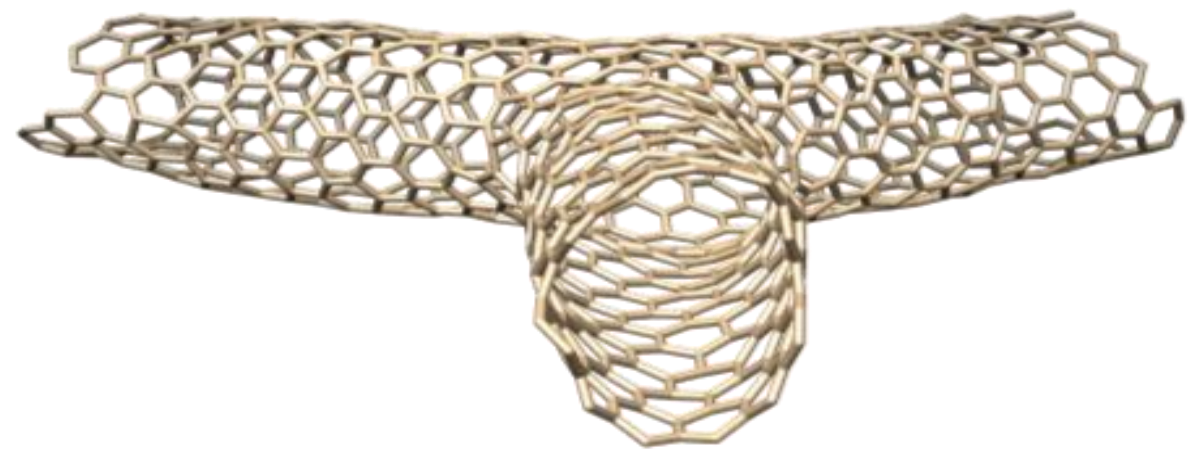

b

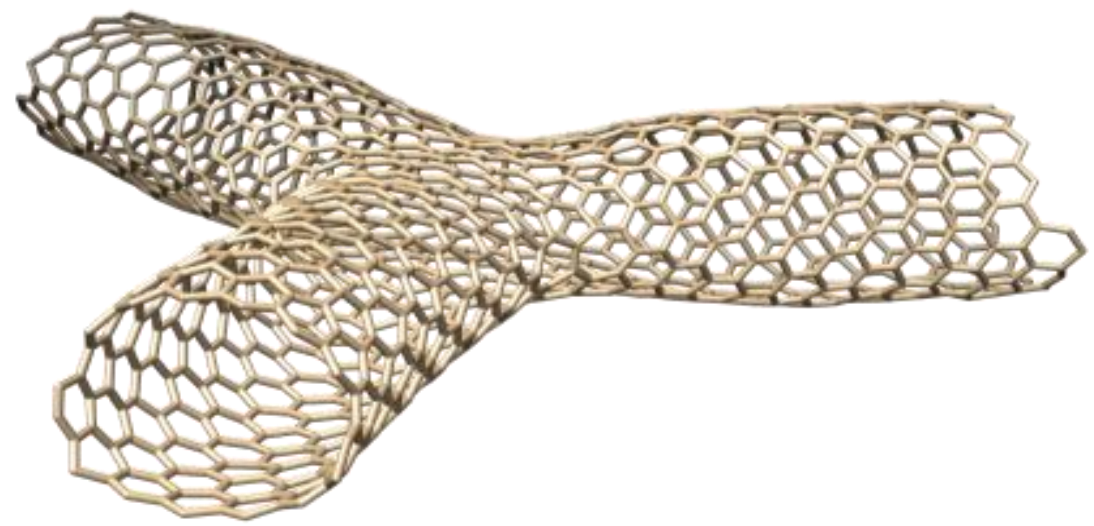

c

Fig. 15: The models of successful zigzag $\mathrm{Y}$-junctions with substrates, after the running of an MD simulation from a distance less than equilibrium $\left(\mathrm{d}_{\mathrm{n}}=3.00 \AA\right)$. a: the result of the MD simulation run on the model in figure 8.a, b: result of the MD simulation run on the model Fig. 8.b , c: result of the MD simulation run on the model of Fig. 8.c.

\section{REFERENCES}

[1]. A. H. Castro Neto, F. Guinea, N. M. R. Peres, K. S. Novoselov, A. K. Geim: The electronic properties of graphene, Reviews of Modern Physics, 81, 2009, 109-165.

[2]. C. G. Rocha, M. H. Rümmeli, I. Ibrahim, H. Sevincli, F. Börrnert, J. Kunstmann, A. Bachmatiuk, M. Pötschke, W. Li, S. A. M. Makharza, S. Roche, B. Büchner,G. Cuniberti: Tailoring the Physical Properties of Graphene, in: Wonbong Choi, Jo-won Lee: „Graphene: Synthesis and Applications", CRC Press, 2011

[3]. A. Uppstu, Electronic properties of graphene from tight-binding simulation, $\mathrm{PhD}$ dissertation, Aalto University, Scool of Science, 2014.

[4]. Yong-Woo Son, Marvin L. Cohen, Steven G. Louie: Energy gaps in graphene nanoribbons, Phys. Rev. Lett. 97, 2006, 216803, 2006, Phys. Rev. Lett. 98, 2007, 089901. 
[5]. X. Li, X. Wang, L. Zhang, S. Lee, H. Dai: Chemically derived, ultrasmooth graphene nanoribbon semiconductors, Science 319 2008, 1229-32.

[6]. Yang, X.; Dou, X.; Rouhanipour, A.; Zhi, L.; Rader, H. \& Muller, K. (2008). Twodimensional graphene nanoribbons. Journal of the American Chemical Society, Vol. 130, No. 13, 4216, ISSN: 0002-7863

[7]. J. Cai, P. Ruffieux, R. Jaafar, M. Bieri, T. Braun, S. Blankenburg, M. Muoth, A. Seitsonen, M. Saleh, X. Feng, K. Mullen and R. Fasel, Atomically precise bottom-up fabrication of graphene nanoribbons. Nature, Vol. 466, No.22, 2010, 470-474.

[8]. Z. Li, W. Zhang, Y. Luo, J. Yang, J.G. Hou, (2009) How Graphene Is Cut upon Oxidation? Journal of the American Chemical Society, 131, 2009, 6320.

[9]. D. Kosynkin, A. Higginbotham, A. Sinitskii, J. Lomeda, A. Dimiev, B. Price, and J. Tour, Longitudinal unzipping of carbon nanotubes to form graphene nanoribbons. Nature, 458, 2009, 872-876.

[10]. L. Jiao, L. Zhang, X. Wang, G. Diankov, H. Dai, Narrow graphene nanoribbons from carbon nanotubes. Nature 458, 2009, 878-880.

[11]. A.J.M. Giesbers, U. Zeitler, S. Neubeck, F. Freitag, K.S. Novoselov, J.C. Maan, Nanolithography and manipulation of graphene using an atomic force microscope, Solid State Communications, 147, 2008, 366369.

[12]. Lishan Weng, Liyuan Zhang, Yong P. Chen, L. P. Rokhinson, Atomic force microscope local oxidation nanolithography of graphene, Appl Phys Lett 93, 20008, 093107.

[13]. L. Tapasztó, G. Dobrik, Ph. Lambin,L.P. Bíró: Tailoring the atomic structure of graphene nanoribbons by scanning tunnelling microscope lithography, Nature Nanotechnology 3, 2008, 397-401.

[14]. G. Dobrik, L. Tapasztó, P. Nemes-Incze, Ph. Lambin, L. P. Biró, Crystallographically oriented high resolution lithography of graphene nanoribbons by STM lithography, Phys. Status Solidi B, 247, 2010, 896-902.

[15]. Sujit S. Datta, Douglas R. Strachan, Samuel M. Khamis, A. T. Charlie Johnson, Crystallographic Etching of Few-Layer Graphene, Nano Lett., 8 , 2008, 1912-1915.

[16]. Lijie Ci, Zhiping, Lili Wang, Wei Gao, Feng Ding, Kevin F. Kelly, Boris I. Yakobson, Pulickel M. Ajayan, Controlled nanocutting of graphene, Nano Res., 1, 2008, 116122.

[17]. Severin N, Kirstein S, Sokolov IM , Rabe JP, Rapid trench channeling of graphenes with catalytic silver nanoparticles, Nano Lett., 9 , 2009, 457-461.

[18]. P. Nemes-Incze, G. Magda, K. Kamarás, L.P. Bíró: Crystallographically selective nanopatterning of graphene on $\mathrm{SiO}_{2}$, Nano Research 3, 2010, 110-116.

[19]. I. László, and I. Zsoldos, Molecular dynamics simulation of carbon nanostructures: The C60 buckminsterfullerene. Physica Status Solidi, B249, 2012, 2616-2619.

[20]. I. László, and I. Zsoldos, Molecular dynamics simulation of carbon nanostructures: The D5h C70 fullerene. Physica, E56, 2014, 422-426.

[21]. I. László, I. Zsoldos, Graphene-based molecular dynamics nanolithography of fullerenes, nanotubes and other carbon structures, Europhysics Letters, 99, 2012, 63001.

[22]. S. S. Han, K. S. Lee, H.M. Lee, Nucleation mechanism of carbon nanotube, Chemical Physics Letters 383, 2004, 321-325.

[23]. L. He, J.Q. Lu, H. Jiang, Controlled CarbonNanotube Junctions Self-Assembled from Graphene Nanoribbons, Small 5, 2009, 28022806.

[24]. D. Fulep, I. Zsoldos, I. Laszlo: Topological and energetic conditions for lithographic production of carbon nanotubes from graphene, Journal of Nanomaterials, Volume 2015 (2015), Article ID 379563

[25]. D. A. Areshkin, C.T. White: Building Blocks for Integrated Graphene Circuits, Nanoletters, 11, 2007, 3253-3259.

[26]. M.P. Allen and D.J. Tildesley, Computer Simulation of Liquids (Clarendon Press, Oxford, 1996).

[27]. D. Frenkel and B. Smit, Understanding Molecular Simulation (Academic Press, San Diego, 1996).

[28]. D. Porezag, T. Frauenheim, T. Köhler, G. Seifert and R. Kaschner, Phys. Rev. B, 51, 1995, 12947-12957.

[29]. L. Verlet, Phys. Rev. 159, 1967 98-103.

[30]. S. Nosé, Mol. Phys. 52, 1984, 255-268.

[31]. W.G. Hoover, Phys. Rev. A, 31, 1985, 16951697.

[32]. D. Fulep, I. Zsoldos, I. Laszlo: Self-organised formation of nanotubes from graphene ribbons: A molecular dynamics study, Materials Research Express 3, Article ID: 105044, 2016.

[33]. Y. Li, Self-assembly of graphene nanoribbon ring on metallic nanowire, Physica E 56, 2014, 277-282.

[34]. Y. Wang, H.F. Zhan, C. Yang, Y. Xiang, Y.Y. Zhang, Formation of carbon nanoscrolls from graphene nanoribbons: A molecular dynamics 
[35]. study, Computational Materials Science 96, 2015, 300-305.

[36]. J.W. Feng, H. M. Ding, Y. Q. Ma, Selfassembly of fullerenes and graphene flake: A molecular dynamics study, Carbon 90, 2015, 34-43.

[37]. J. Wallace, L. Shao, Defect-induced carbon nanoscroll formation, Carbon 91, 2015, 96102.
[38]. W. Li, X. Zheng, B. Liu, X. Sun, T. Wang, J. Zhang, Y. Yan, A novel method for designing carbon nanostructures: Tailoring-induced selfscrolling of graphene flakes, Carbon 89, 2015, 272-278

[39]. W. Sun, Q. Zheng, A. Yu, Interaction forces between carbon nanospheres: A molecular dynamics simulation study, Chemical Engineering Science, 121, 2015, 23-31. 